\title{
Erratum
}

\section{Copper-Catalyzed Sulfonylation of Arylboronic Acids in Ionic Liquids}

M. Lakshmi Kantam,* B. Neelima, B. Sreedhar, Rajashree Chakravarti Synlett 2008, 1455.

In Table 2, entry 7, the R group was given as Me but should instead be $p$-Tol. The correct entry is shown below.

Table 2 Cross-Coupling Reaction between Various Arylboronic Acids and Sulfinate Salts ${ }^{\mathrm{a}}$

\begin{tabular}{llll}
\hline Entry Arylboronic acid & $\mathrm{R}$ & Product & $\begin{array}{l}\text { Isolated } \\
\text { yield (\%) }\end{array}$ \\
\hline $\mathrm{B}(\mathrm{OH})_{2}$ & $p$-Tol &
\end{tabular}

${ }^{\mathrm{a}}$ Reaction conditions: $\mathrm{Cu}(\mathrm{OAc})_{2}(0.013 \mathrm{~g}, 10 \mathrm{~mol} \%)$, [bmim][OTf] $(1 \mathrm{~mL})$, arylboronic acid $(1 \mathrm{mmol})$, sodium sulfinate salt $(1 \mathrm{mmol})$, r.t. for $12 \mathrm{~h}$.

${ }^{\mathrm{b}}$ Isolated yield after fifth cycle. 